\title{
Síndrome metabólico y resistencia insulínica en pacientes con Diabetes Mellitus tipo 1: prevalencia
}

\section{Metabolic syndrome and insulin resistance in patients with Diabetes Mellitus type 1: prevalence}

doi) http:// dx.doi.org/10.35954/SM2019.38.1.3

\author{
Lorena Bruno González a (1) https://orcid.org/0000-0001-5678-7971 \\ Claudia Pérez Buenahora b (D) https://orcid.org/0000-0003-2759-2694
}

(a) Departamento de Medicina. Hospital Pasteur. Montevideo. Uruguay.

(b) División Epidemiología - Vigilancia de Fronteras. Ministerio de Salud Pública. Montevideo. Uruguay.

\section{RESUMEN}

El Síndrome Metabólico es una entidad clínica compleja, definida por la presencia de un conjunto de factores de riesgo metabólicos que asociados a la Insulinorresistencia, aumentan el riesgo de afecciones cardiovasculares en los individuos no diabéticos y en los pacientes con Diabetes Mellitus tipo 2.

La presencia de Síndrome Metabólico y sus componentes se ha descrito también en los pacientes con Diabetes Mellitus Tipo 1, lo que para algunos definiría el concepto de Diabetes Doble. Por tal motivo, el objetivo planteado para este trabajo consiste en determinar la relación existente entre los componentes del Síndrome Metabólico y la cuantificación de la Insulinorresistencia en pacientes con Diabetes Mellitus tipo 1.

La identificación en la práctica clínica de un grupo de pacientes con Diabetes Mellitus tipo 1, con fenotipo de sobrepeso/obesidad, nos conduce a pensar que estamos frente a una población específica, con mayor riesgo de complicaciones micro y macrovasculares. Decidimos investigar la prevalencia de síndrome metabólico en pacientes con Diabetes Mellitus tipo 1 que se asisten y controlan en la Unidad Docente Asistencial de Diabetes, ubicada dentro del Hospital Pasteur, durante el período Abril-Junio 2018; considerando que la profundización en el tema generará un aporte al conocimiento en un área inexplorada en nuestro medio y relevante para mejorar el enfoque clínico y el abordaje terapéutico de estos pacientes.

PALABRAS CLAVE: Diabetes Mellitus Tipo 1; Diabetes Mellitus Tipo 2; Resistencia a la Insulina; Síndrome Metabólico.

\section{ABSTRACT}

The Metabolic Syndrome is a complex clinical entity, defined by the presence of a set of metabolic risk factors that, associated with Insulin Resistan$\mathrm{ce}$, increase the risk of cardiovascular diseases in non-diabetic individuals and in patients with Type 2 Diabetes Mellitus. The presence of Metabolic Syndrome and its components has also been described in patients with Type 1 Diabetes Mellitus, which for some would define the concept of Double Diabetes. For this reason, the objective set for this paper is to determine the relationship between the components of the Metabolic Syndrome and the quantification of Insulin Resistance in patients with Type 1 Diabetes Mellitus. The identification in the clinical practice of a group of patients with Diabetes Mellitus type 1, with phenotype of overweight / obesity, leads us to think that we are facing a specific population, with greater risk of micro and macrovascular complications. We decided to investigate the prevalence of the metabolic syndrome in patients with type 1 diabetes mellitus 
that are attended and controlled in the Diabetes Care Teaching Unit, located within the Pasteur Hospital, during the period April-June 2018; considering that a deeper exploration of the subject will improve the contribution to our knowledge in an unexplored area in our environment and it shall be significant in order to improve the clinical and therapeutic approach towards these patients.

KEY WORDS: Diabetes Mellitus Type 1; Diabetes Mellitus Type 2; Insulin Resistance; Metabolic Syndrome.

\section{INTRODUCCIÓN}

\section{Definición de Diabetes Mellitus (DM)}

Desorden metabólico de múltiples etiologías, resultante de alteraciones en la secreción y/o acción de la insulina, que conduce a hiperglicemia crónica implicando alteraciones en el metabolismo de los hidratos de carbono, proteínas y lípidos (1).

\section{Criterios diagnósticos de DM}

1) glicemia en ayunas $\geq 126 \mathrm{mg} / \mathrm{dl}$,

2) glicemia al azar $\geq 200 \mathrm{mg} / \mathrm{dl}$ con síntomas,

3) glicemia medida 2 horas posterior a carga oral con $75 \mathrm{gr}$ de glucosa (prueba de tolerancia oral a la glucosa o PTOG) $\geq 200 \mathrm{mg} / \mathrm{dl}$,

4) hemoglobina glicosilada $(\mathrm{Hb} \mathrm{A} 1 \mathrm{c}) \geq 6,5 \%$ con metodología NGSP. En nuestro medio la $\mathrm{Hb}$ A1c no está estandarizada como método diagnóstico. Los criterios 1 y 3 deben verificarse en dos oportunidades $(1,2)$.

\section{Clasificación}

La DM se clasifica hoy en 4 categorías:

1) tipo 1 (DM1): destrucción inmunitaria de célula beta que conduce a déficit absoluto de insulina,

2) tipo 2 (DM2): pérdida progresiva de la secreción pancreática de insulina en el contexto de resistencia insulínica (RI),

3) diabetes gestacional: es aquella diabetes que se diagnostica en el segundo o tercer trimestre de embarazo en una paciente no conocida como diabética, 4) tipos específicos de diabetes: monogénica (MODY), diabetes neonatal, enfermedades del páncreas exocrino (fibrosis quística), diabetes secundaria inducida por fármacos (glucocorticoides, antirretrovirales, diabetes post-trasplante por otros inmunosupresores)(2).

La clasificación es relevante a la hora de determinar la terapéutica; sin embargo, tanto diabetes tipo 1 como tipo 2 son patologías heterogéneas (en ambas, existen múltiples factores ambientales y genéticos que determinan afectación de la célula beta pancreática, afectando la masa y/o la funcionalidad celular, conduciendo a manifestaciones clínicas asociadas a hiperglicemia) cuya presentación clínica puede ser variada y conducir a dificultades para catalogarlos al momento del diagnóstico, siendo más certera la clasificación en la evolución (2).

Esta clasificación tradicional parece ser insuficiente para reflejar la heterogeneidad genotípica-fenotípica que puede existir. Nuevas propuestas de clasificación toman en consideración: parámetros clínicos (edad al diagnóstico, Índice de Masa Corporal (IMC)); la cuantificación de marcadores metabólicos (péptido $\mathrm{C}, \mathrm{HbA1c}$, determinación del índice homeostasis model assessment (HOMA -IR)) e inmunológicos (determinación de anticuerpos - específicamente anticuerpo contra en ácido glutámico decarboxilasa (GADA)). De lo analizado, se podrían agrupar los pacientes en 5 subgrupos (3):

1) $\mathrm{DM}$ de inicio temprano: $6,4 \%$ IMC bajo, pobre control metabólico, baja secreción de insulina y GADA positivo: DM autoinmune severa;

2) DM de inicio temprano: IMC bajo, pobre control metabólico, baja secreción de insulina y GADA negativo: 17,5\% DM insulino deficiente severa;

3) DM con IR e IMC alto: 15,3\%. DM insulino resistente;

4) Obesos pero sin IR 21,6\%: DM leve asociada a obesidad;

5) DM diagnosticada a mayor edad, escasas alteraciones metabólicas: 39,1\%. DM leve asociada a edad. Algunos autores plantean la necesidad de afinar y precisar la tipificación de la DM, usando otros tests de laboratorio. Se destaca la importancia de contar con herramientas genéticas, inmunes y metabólicas como predictores de progresión. La posibilidad de 
predecir respuestas terapéuticas asegura un enfoque más personalizado (4).

Volviendo a la clasificación clásica de la DM:

1) La DM1 está presente en $5-10 \%$ de todos los casos de DM; presenta fuerte asociación con el sistema mayor de histocompatibilidad, sistema del antígeno leucocitario humano (HLA) y en su gran mayoría (diabetes inmuno-mediada). Se asocia a la presencia de uno o más de los siguientes autoanticuerpos: antiglutamato decarboxilasa 65 (GADA 65), anti insulina, anti tirosina fosfatasa IA-2 y IA20 y anti transportador de zinc (ZnT8). Factores como la edad de presentación, el número de auto anticuerpos, así como el título y especificidad de los mismos determinarán la progresión de la enfermedad. La edad de presentación clínica más habitual es en la niñez o juventud; sin embargo puede presentarse en cualquier momento de la vida. Los factores ambientales que gatillan la destrucción autoinmune de la célula beta son variados y en su mayoría desconocidos. La presencia de obesidad, es un hecho cada vez más frecuente sin embargo no debería excluir este diagnóstico. En una minoría de pacientes con DM1 no se logra demostrar la presencia de anticuerpos (diabetes tipo 1 idiopática). En cualquier caso, la tendencia a la cetosis está presente y la terapia insulínica es determinante para la supervivencia (2).

2) La DM2 representa $90-95 \%$ de los casos de DM. En su génesis se encuentra el déficit relativo, más que absoluto de insulina en el contexto de resistencia periférica a la insulina. Los niveles de insulina pueden ser normales o altos, pero insuficientes para superar la RI. La mayoría de los pacientes tienen sobrepeso/obesidad, lo cual favorece la RI. La cetosis suele verse en general asociada a stress o infección; es probable que al menos inicialmente estos pacientes no requieran insulina para la supervivencia (2).

\section{Definición de Síndrome Metabólico (SM)}

Se define como la confluencia de varios factores de riesgo metabólicos en un sólo individuo. Son componentes del SM: obesidad abdominal, dis- lipemia aterogénica, presión arterial elevada, intolerancia a la glucosa, RI, estado protrombótico y proinflamatorio. Para definir SM debemos contar con al menos tres de los siguientes criterios: obesidad abdominal (circunferencia de cintura > $102 \mathrm{~cm}$ en hombres y $>88 \mathrm{~cm}$ en mujeres), triglicéridos $\geq 150 \mathrm{mg} / \mathrm{dL}$, HDL colesterol $<40 \mathrm{mg} / \mathrm{dl}$ en hombres y $<50 \mathrm{mg} / \mathrm{dl}$ en mujeres, glicemia en ayunas $\geq 110 \mathrm{mg} / \mathrm{dl}$ y la hipertensión (HA): presión arterial $\geq 130 / 85$ (5). Para nuestro trabajo consideraremos HA según criterio del $8^{\circ}$ Joint National Comittee (PA > 140/90 para diabéticos) (6).

\section{Definición De Resistencia Insulínica (RI)}

Se define como un estado metabólico en el cual la respuesta tisular a la insulina es menor a la esperada para la insulina disponible. Tiene estrecha relación con el SM; alteraciones metabólicas e inflamatorias a distintos niveles (molecular, celular y hemodinámico) se asocian a Rl y a adiposidad visceral. Si bien se relaciona comúnmente a DM2, puede estar presente en DM1. Se asocia a mal control metabólico y constituye un factor de riesgo independiente tanto de micro como de macroangiopatía $(7-9,12)$. La RI se caracteriza clínicamente por un alto requerimiento insulínico (> $1 \mathrm{u} / \mathrm{K} /$ día). El método de referencia para calcular RI es el clamp euglicémico hiperinsulinémico, el cual ha demostrado ser una herramienta fiable para distinguir RI en DM1, incluso en distintas etnias. No se aplica en la práctica clínica, siendo una herramienta utilizada en protocolos de investigación clínica; es así que se ha desarrollado otra forma de cuantificarla de forma no invasiva a través del cálculo de captación de glucosa (CG) $(10,11,13)$ con la fórmula propuesta por The Pittsburg Epidemiology of Diabetes Complications (14), validada con el clamp euglicémico hiperinsulinémico $(8,12)$, según la cual:

$C G=24,3-(12,22 x$ índice cintura/cadera) $-(3,29 x$

$$
\text { HA) - 0,57 } \times \text { HbA1c) }
$$

Esta fórmula considera HA con puntaje 0 si el paciente no es hipertenso y puntaje 1 si tiene historia personal de HA y se utilizará en pacientes con $\mathrm{HbA} 1 \mathrm{c}<11,4 \%$. 
El punto de corte que determina la captación de glucosa no es unánime. Para este trabajo se tomó como punto de corte el valor 8,77 . Valores CG < 8,77 indican mayor IR y valores $\geq 8,77$ indican menor IR $(7,8)$.

\section{Diabetes Doble (DD)}

El término "diabetes doble" (DD), describe los pacientes con DM1 asociados a SM (15-17). Acuñado en 1991 (18), no es un término universalmente aceptado. Intenta englobar un grupo de pacientes con riesgo metabólico especialmente alto, independiente del control metabólico $(15,16)$. La ganancia de peso vinculada a los cambios alimentarios (factores culturales, sedentarismo), conjuntamente con el tratamiento intensificado de la DM1, estarían relacionados a un aumento del riesgo cardiovascular $(17,19,20)$. La estimación de RI en DM1 podría tener implicancias terapéuticas en términos de abordaje intensivo del control de los factores de riesgo cardiovascular implicados en el SM (21); el uso de metformina es una herramienta a valorar en este subgrupo de pacientes (19).

\section{ANTECEDENTES}

EI SM asociado a DM1 presenta una alta prevalencia a nivel mundial, que en las distintas publicaciones oscila entre $18,5 \%$ y $42 \%$ (8). Estas diferencias se explican por las distintas regiones geográficas y los distintos criterios para definir la entidad SM. En una distribución regional, encontramos en Sudamérica las siguientes prevalencias: Chile 25\% (22) y 26,6\% (8); Brasil $45 \%$ (definiendo SM con criterio WHO) (23).

En Centroamérica, los datos disponibles son de México, con prevalencias publicadas de 18,5\% (24) y $25 \%$ (16). En Europa se reporta $32 \%$ de prevalencia en España (19), 26,8\% en Rumania (25) y $25 \%$ en Alemania (15).

\section{Situación del Uruguay, antecedentes}

En noviembre de 1998, se realizó el primer estudio epidemiológico nacional que permitió evaluar la prevalencia de sobrepeso y obesidad. Se trabajó con una muestra de 900 individuos, mayores de 18 años, representativa de Uruguay urbano, se calculó Índice de Masa Corporal (IMC) por autorreporte de peso y talla. Los resultados obtenidos de esta Primera Encuesta Nacional de Sobrepeso y Obesidad (ENSO I) y su relación con las comorbilidades de la obesidad, fueron los siguientes: $51 \%$ de la población se encontraba en sobrepeso (IMC $\geq 25$ ) de este porcentaje, el $17 \%$ se encontraba en diferentes grados de obesidad (IMC $\geq 30$ ). Los hombres presentaban más sobrepeso e igual obesidad que las mujeres, las mujeres igualaban a los hombres en obesidad a partir de los 55 años y luego los sobrepasaban. Se observó que el sobrepeso y obesidad se presentaba en mayores de 55 años, y era mayor en los estratos sociales bajos. También se observó una relación inversa entre IMC y educación. Con respecto al IMC y sedentarismo, $50 \%$ de la población con normopeso declaró realizar ejercicio frecuentemente, mientras que tan solo el $30 \%$ de la población obesa realizaba algún tipo de ejercicio. Con respecto a las comorbilidades: $10 \%$ de los encuestados sabían que tenían "colesterol elevado", 21\% HA, 4,7 refirió DM conocida, de los cuales $4 \%$ era DM 2. Ya en el año 2000 se afirmaba que Uruguay padecía una verdadera epidemia de obesidad que incrementaba las enfermedades cardiovasculares y la DM, y las causas de esta epidemia obedecían a la interacción de factores ambientales determinados por el estilo de vida moderno, sedentarismo y alta ingesta de grasas (26). En Montevideo, en el año 2004, se realiza el primer estudio de prevalencia de DM, en población adulta de entre 20 y 79 años de edad, de ambos sexos y todas las razas. Los resultados mostraron una prevalencia de $6,4 \%$ de diabéticos conocidos y $1,6 \%$ de diabéticos no conocidos, totalizando $8 \%$ de la población adulta. Con respecto a las glicemias alteradas fueron de 2,8\% según criterios OMS 1997 y 8,2\% según criterios ADA 2004. En los resultados de un total de 39 diabéticos conocidos, 3 correspondían al tipo 1 y 36 de tipo 2 . Se observó un predominio de sexo femenino que representó un $64 \%$ del total. En cuanto a la edad, se observó un aumento de casos 
a partir de los 50 años (27).

En mayo del año 2006, se realiza la Segunda Encuesta Nacional de Sobrepeso y Obesidad (ENSO II), en individuos entre 18 y 65 años, publicada en el año 2009. La misma concluyó que 1 de cada 2 adultos uruguayos padecía sobrepeso $\mathrm{u}$ obesidad y 1 de cada 5 era obeso. Es así que en el año 2006 se confirmaría que estos hallazgos determinaban un alto riesgo sanitario para la población general y económico para nuestro sistema nacional de salud. Los resultados obtenidos fueron los siguientes: $46 \%$ de la población adulta se encuentra en rango de normopeso IMC $<25 \mathrm{~kg} / \mathrm{m}^{2}, 34 \%$ presenta sobrepeso, IMC entre $25-29 \mathrm{~kg} / \mathrm{m}^{2}$ y $20 \%$ obesidad IMC $>30 \mathrm{~kg} / \mathrm{m}^{2}$.

Las mujeres presentaban mayor obesidad que los hombres a partir de los 55 años, el sobrepeso y la obesidad aumentaba linealmente con la edad, presentando un notorio incremento a partir de los 35 años, alcanzando niveles muy altos en los mayores de 45 años. El rango de sobrepeso es elevado en todos los estratos socioeconómicos, en cambio en la franja de obesidad se observaron diferencias entre los estratos socioeconómicos alto y medio-alto, $12,2 \%$, versus medio-bajo y bajo $18 \%$.

Se sigue manteniendo una relación inversa entre el nivel educacional y el IMC. Si bien las cifras del ENSO I no son comparables con los datos objetivos del ENSO II, debido a que en ENSO I los datos de peso y talla eran autoreferenciados por los individuos participantes, así mismo no existieron diferencias significativas en relación con la proporción de sobrepeso y obesidad por sexo. La década entre los 35 y 45 años se indicaba como un punto de inflexión abrupta en el aumento de la obesidad en Uruguay.

Se observó que el nivel socioeconómico y cultural influía notoriamente en la incidencia de la obesidad que predominaba en estratos sociales bajo y medio. Observaron que la obesidad y sus comorbilidades aumentan en forma lineal con la edad, el SM, la DM 2 y la HA, todas enfermedades relacionadas estrechamente a la obesidad, alcanzando niveles muy elevados después de los 45 años. La prevalencia de obesidad se incrementaba linealmente década tras década, desde los 35 hasta los 65 años y más, es decir, que la obesidad se veía aumentada en la población que asocia más factores de riesgo vascular (26). En marzo del año 2009, Uruguay presentó los resultados de la Primera Encuesta Nacional de Factores de Riesgo de Enfermedades Crónicas No Transmisibles, realizada en el año 2006. En la misma se estudió una muestra de población adulta (25-64 años de edad), de ambos sexos y se describieron algunos factores de riesgo de las Enfermedades Crónicas No Transmisibles (ECNT). Entre ellos destacamos: según IMC, el $57 \%$ de la población presentaba sobrepeso u obesidad, con respecto a la circunferencia de cintura (CC) en el $21 \%$ de los hombres era $\geq 102$ $\mathrm{cm}$ y el $39 \%$ de las mujeres era $\geq 88 \mathrm{~cm}$. Con respecto a la prevalencia de la $\mathrm{HA}$, se encontró un $10 \%$ en individuos entre 25 y 34 años y hasta un $70 \%$ entre los 55 a 64 años de edad (28).

En la Segunda Encuesta Nacional de Factores de Riesgo de ECNT realizada en el año 2013, la prevalencia de sobrepeso y obesidad en personas de 25 a 64 años fue de $64,9 \%$, es decir, que casi 2 de cada 3 personas registraron sobrepeso/obesidad, observándose diferencias significativas entre jóvenes y adultos: $37,7 \%$ entre los jóvenes, respecto a $68,3 \%$ entre los adultos. A mayor edad, mayor sobrepeso/ obesidad. Cabe destacar, que en esta encuesta se amplió el universo de estudio, contándose por primera vez con información de la población entre 15 y 24 años de edad, residentes en localidades urbanas de Uruguay. El promedio de IMC para el año 2006 (1 ${ }^{\text {a }}$ Encuesta Nacional) para ambos sexos era de 26,4 $\mathrm{Kg} / \mathrm{m}^{2}$, no existiendo diferencias significativas entre hombres y mujeres; para el año 2013 era de $27,7 \mathrm{Kg} /$ $\mathrm{m}^{2}$ para ambos sexos. Con respecto a la CC de riesgo, la prevalencia de la misma fue de $41,0 \%$ para ambos sexos, existiendo diferencias significativas entre ambos sexos: $51,1 \%$ de las mujeres posee una CC de riesgo, mientras que en los hombres es de $29,9 \%$. La prevalencia de HA ( $\geq 140 / 90$ ) fue de aproximadamente 3 de cada 10 personas, alcanzando a más de $1 / 3$ de la población de 25 a 64 años, siendo mayor en el sexo masculino adulto. Se observó además que 
esta prevalencia es 9 veces mayor en el grupo de 55 a 64 años, respecto a la registrada en el grupo más joven (15 a 24 años), tal cual lo indica la evidencia. Además, casi 6 de cada 10 personas presentaron cifras "alteradas" de HA, al tiempo que manifestaron no estar en tratamiento alguno. Esta cifra fue mayor entre los hombres.

Para la estimación de la prevalencia de DM se consideraron las medidas de glicemia $\geq 126 \mathrm{mg} / \mathrm{dl}$ (obteniendo la muestra por punción digital, los valores de sangre capilar fueron calibrados a valores plasmáticos), se incluyeron a las personas encuestadas que refirieron ser diabéticas y estar en tratamiento medicamentoso. La prevalencia de DM se ubicó en el orden de $6 \%$ para la población de 15 a 64 años, siendo mayor en la población adulta ( 25 a 64 años) en relación a la población joven (15 a 24 años). No se registraron diferencias estadísticamente significativas entre hombres y mujeres. La prevalencia de glicemia de ayuno alterada (GAA) fue de $3 \%$ de la población entre 15 y 64 años, resultando cifras menores entre los más jóvenes. Si se considera a la población con DM y/o GAA, la prevalencia se ubicaba en el entorno del 9\% para la población de 15 a 64 años, afectando a 1 de cada 10 personas entre 25 y 64 años. La cifra es claramente menor entre los más jóvenes: $2 \%$.

Con respecto a la colesterolemia elevada, más de 1 cada 5 personas entre 25 y 64 años presentaba colesterolemia elevada ( $\geq 200 \mathrm{mg} / \mathrm{dl}$ ), aproximadamente un 20\%, en el año 2013.

Concluimos que, en Uruguay, la última Encuesta Nacional de Factores de Riesgo para Enfermedades No Transmisibles del año 2013 mostró una prevalencia de estos factores por encima de $20 \%$, algunos de ellos componentes del llamado SM (29).

\section{OBJETIVOS}

\section{Objetivo general}

Determinar la relación existente entre los componentes del SM y la cuantificación de la RI, en pacientes con DM 1 que se controlan en la Unidad Docente Asistencial (UDA) de Diabetes del Hospital Pasteur, Abril-Junio 2018.

\section{Objetivos específicos}

1) Caracterizar la población de estudio según variables demográficas;

2) Determinar la prevalencia de los criterios que componen el SM, en DM 1;

3) Cuantificar la resistencia insulínica a través de la CG;

4) Establecer la relación entre RI y SM;

5) Correlacionar el control metabólico (dosificación de $\mathrm{Hb} \mathrm{A1c)}$ con la CG.

\section{DISEÑO METODOLÓGICO}

\section{Tipo de estudio, población y método}

Nuestro trabajo consistió en un estudio descriptivo, transversal o de prevalencia, que se realizó en pacientes con diagnóstico de DM tipo 1 , que se asisten y controlan en la UDA de Diabetes del Hospital Pasteur. Trabajamos con la totalidad de pacientes y sus historias clínicas que constituyó nuestro universo, los mismos fueron mayores de 15 años de edad, de ambos sexos y con su consentimiento informado. Se definió como caso al paciente con diagnóstico de DM 1, entendiendo por tal a aquel con DM que requiere insulinoterapia desde su debut para su supervivencia.

A los casos (número de pacientes) se les determinó la presencia de SM según criterio del National Cholesterol Education Program (NCEP/ATPIII).

Para la búsqueda bibliográfica por tema usamos como descriptores: Diabetes Mellitus Tipo 1, Síndrome Metabólico e Insulinorresistencia. En referencia a los años de publicación, se abarcaron los últimos 12 años y se buscó en los idiomas: español, inglés y portugués, en las bases de datos MedLine, Lilacs y Bireme.

\section{Variables}

Cuantitativas: edad, tiempo de evolución de la DM 1, Circunferencia de Cintura, Perímetro de Cadera, valor de HbA1c, Triglicéridos, HDL.

Cualitativas: Procedencia, ICC, HA.

Para definir HA se tomó en cuenta el antecedente personal de ser hipertenso (PA > 140/90) según cri- 
terio del Joint National Comittee VIII, o estar tomando medicación antihipertensiva.

La circunferencia de cintura (CC) se midió con una cinta métrica flexible en forma horizontal a nivel de la zona hendida entre el último arco costal y la cresta ilíaca, con el paciente de pie y la pared abdominal relajada, al final de una respiración normal.

El perímetro de cadera (PC) se determinó con la misma cinta métrica, en forma horizontal a nivel más prominente de los glúteos con el sujeto de pie.

Con las variables anteriores calculamos el índice cintura cadera (ICC)

La HbA1c se midió con técnica de laboratorio certificada por International Federation of Clinical Chemistry (IFCC) y National Glycohemoglobin Standardization Program (NGSP), método inmunoturbidimétrico. El colesterol HDL y los triglicéridos se midieron con las técnicas de laboratorio del Hospital Pasteur (método enzimático colorimétrico).

A todos los pacientes se les calculó la RI, según fórmula:

\section{$C G=24,3-12,22 \times$ índice cintura/cadera - 3,29 x}

\section{HTA - 0,57 x HbA1c}

Se comparó los hallazgos de CG en el grupo sin SM vs el grupo con al menos un componente del SM.

Todos los resultados de la paraclínica se obtuvieron de las historias clínicas de los pacientes.

\section{Fuentes}

Utilizamos fuentes primarias y secundarias.

Fuente primaria: mediciones antropométricas (cc y pc) mediante entrevista.

Fuente secundaria: historia clínica del paciente, de donde obtuvimos tiempo de evolución de la enfermedad, antecedente de HA, los registros previos de: mediciones peso y talla y la paraclínica (TG, HDL, $\mathrm{HbA1c}$ ) que constituyeron algunas de las variables de nuestra investigación.

\section{Métodos e instrumento de recolección de datos:}

1) Se realizó registro de las historias clínicas $(\mathrm{HC})$;

2) Se realizó entrevista estructurada a los pacientes;

3) Las autoras realizaron mediciones antropométricas (peso, talla y perímetro de cintura).

\begin{tabular}{l}
$\quad \begin{array}{l}\text { Síndrome Metabólico y Resistencia Insulínica en } \\
\text { pacientes con Diabetes Mellitus 1: prevalencia } \\
\text { UDA Diabetes, Hospital Pasteur 2018 }\end{array}$ \\
\begin{tabular}{l}
$\quad$ No orden: \\
\hline Fecha:
\end{tabular} \\
\hline Nombre y apellido: \\
\hline C.I.: \\
\hline Procedencia (Montevideo/Interior): \\
\hline Sexo (F/M): \\
\hline Edad (años): \\
\hline \\
\hline Tiempo de evolución de DM (años): \\
\hline Peso(kg): \\
\hline Talla (m): \\
\hline IMC: \\
\hline Circunf cintura (CC) (cm): \\
\hline Perímetro cadera (PC) (cm): \\
\hline ICC: \\
\hline HbA1c (\%): \\
\hline TG (mg/dl): \\
\hline HDL (mg/dl): \\
\hline HA (SI/NO): \\
\hline OBSERVACIONES: \\
\hline Encuestador: \\
\hline
\end{tabular}

Figura 1. Formulario de recolección de datos.

Como instrumento de recolección de estos datos, utilizamos un formulario semicerrado (figura 1).

Aplicación de tests estadísticos: como medidas de resumen se utilizaron las medidas de tendencia central y de dispersión, según corresponda, Test de $T$ y Test de $Z$ dependiendo el cruce de variables, cuantitativas continuas y chi cuadrado para las variables cualitativas.

\section{RESULTADOS}

De un total de 64 pacientes, el $62 \%$ procede de Montevideo (figura 2). El 55\% pertenece al sexo masculino (figura 3). La edad promedio es de $33 \pm$ 15 años (DE), rango de 16 a 83. De éstos, $31 \%$ (20 casos) son adolescentes cuyas edades están comprendidas entre 15 y 19 años (según definición de Organización Mundial de la Salud, OMS). 42\% (27 casos) son adultos jóvenes (edades entre 20 y 44) (figura 4). Con respecto al tiempo de evolución de la DM, la media fue de $12 \pm 11$ años (DE); en el $59 \%$ el tiempo de evolución es menor a los 10 años (figura 


\begin{tabular}{|c|c|c|c|c|c|c|c|}
\hline \multicolumn{2}{|c|}{ Montevideo } & \multicolumn{2}{c|}{ Interior } & \multicolumn{2}{c|}{ Sin dato } & \multicolumn{2}{c|}{ Total } \\
\hline F.A & F.R (\%) & F.A & F.R (\%) & F.A & F.R (\%) & F.A & F.R (\%) \\
\hline 40 & 62 & 19 & 30 & 5 & 8 & 64 & 100 \\
\hline
\end{tabular}

Figura 2. Distribución de número de casos de DM1 según procedencia.

\begin{tabular}{|c|c|c|c|c|c|}
\hline \multicolumn{2}{|c|}{ Masculino } & \multicolumn{2}{c|}{ Femenino } & \multicolumn{2}{c|}{ Total } \\
\hline $\mathrm{N}^{\circ}$ casos & F.relativa (\%) & $\mathrm{N}^{\circ}$ casos & F.relativa (\%) & $\mathrm{N}^{\circ}$ casos & F.relativa (\%) \\
\hline 35 & 55 & 29 & 45 & 64 & 100 \\
\hline
\end{tabular}

Figura 3. Distribución de número de casos de DM1 según sexo.

\begin{tabular}{|c|c|c|c|}
\hline Rango etario (años) & Masculino & Femenino & Total (FA) \\
\hline adolescente (15-19) & 13 & 7 & 20 \\
\hline Adulto joven ( 20-44) & 10 & 17 & 27 \\
\hline Adulto maduro (45-64) & 10 & 5 & 15 \\
\hline Adulto mayor (Z65) & 2 & 0 & 2 \\
\hline TOTAL & 35 & 29 & 64 \\
\hline
\end{tabular}

Figura 4. Distribución de número de casos de DM1 según rango etario, por sexo.

\begin{tabular}{|c|c|c|c|}
\hline $\begin{array}{c}\text { Tiempo de evolución } \\
\text { de la DM (años) }\end{array}$ & Masculino & Femenino & FR (\%) \\
\hline $1-10$ & 22 & 16 & 59 \\
\hline $11-21$ & 7 & 6 & 20 \\
\hline $22-31$ & 3 & 3 & 9 \\
\hline $32-41$ & 2 & 3 & 7 \\
\hline $42-51$ & 0 & 1 & 2 \\
\hline Sin datos & 1 & 0 & 2 \\
\hline TOTAL & 35 & 29 & 100 \\
\hline
\end{tabular}

Figura 5. Distribución de número de casos de DM1 según tiempo de evolución de la DM, por sexo.

5). El $62 \%$ de la población presenta normopeso; el $38 \%$ restante presenta sobrepeso/obesidad y son adultos jóvenes (figura 6 ).

Presenta perímetro de cintura de riesgo el $20 \%$ de la población estudiada (de los cuales $75 \%$ son mujeres adultas jóvenes). Si analizamos ambas variables, observamos que el sobrepeso u obesidad que registramos no es necesariamente a expensas de obesidad centroabdominal (figuras 7 y 8 ).

Presenta HDL de riesgo el $25 \%$ de la población, todos adultos jóvenes (69\% mujeres y $31 \%$ hombres). Encontramos un $16 \%$ de pacientes con hipertrigliceridemia, siendo el $75 \%$ hombres adultos jóvenes.

En relación a HA, $10 \%$ la presenta; de éstos, $67 \%$

\begin{tabular}{|c|c|c|c|c|c|}
\hline \multirow{2}{*}{$\begin{array}{c}\text { RANGO } \\
\text { ETARIO }\end{array}$} & \multicolumn{3}{|c|}{ IMC } & \multirow{2}{*}{$\begin{array}{c}\text { SIN } \\
\text { DATO }\end{array}$} & \multirow{2}{*}{ TOTAL } \\
\cline { 2 - 4 } & $\mathbf{2 5}$ & $\mathbf{2 5 - 2 9}$ & $\mathbf{3} \mathbf{3 0}$ & \\
\hline & FA & FA & FA & FA & FA \\
\hline $15-19$ & 9 & 6 & 0 & 5 & 20 \\
\hline $20-44$ & 15 & 2 & 6 & 4 & 27 \\
\hline $45-64$ & 8 & 4 & 1 & 2 & 15 \\
\hline$\geq 65$ & 1 & 0 & 1 & 0 & 2 \\
\hline TOTAL & 33 & 12 & 8 & 11 & 64 \\
\hline
\end{tabular}

Figura 6. Distribución de número de casos de DM1 según IMC, por rango etario.

\begin{tabular}{|c|c|c|c|}
\hline $\begin{array}{c}\text { Perímetro de } \\
\text { cintura }\end{array}$ & $\begin{array}{c}\text { Masculino } \\
\text { (FA) }\end{array}$ & $\begin{array}{c}\text { Femenino } \\
\text { (FA) }\end{array}$ & $\begin{array}{c}\text { Total } \\
\text { (FA) }\end{array}$ \\
\hline De riesgo & 2 & 8 & 10 \\
\hline No Riesgo & 26 & 14 & 40 \\
\hline Sin dato & 7 & 7 & 14 \\
\hline TOTAL & 35 & 29 & 64 \\
\hline
\end{tabular}

Figura 7. Distribución de número de casos de DM1 según perímetro de cintura, por sexo.

\begin{tabular}{|c|c|c|}
\hline Rango etario & Masculino & Femenino \\
\hline 15-19 adolescente & 1 & 0 \\
\hline 20-44 adulto joven & 1 & 6 \\
\hline 45-64 adulto maduro & 0 & 2 \\
\hline$\geq 65$ adulto mayor & 0 & 0 \\
\hline Sin datos & 7 & 7 \\
\hline
\end{tabular}

Figura 8. Distribución de número de casos de DM1 según perímetro de cintura de riesgo, por rango etario.

son adultos maduros y mayores (entendiendo por adultos maduros a aquellos cuyas edades estén comprendidas entre 45 y 64 años, y adultos mayores aquellos con edades $\geq$ a 65 años). El restante 33\% es menor de 44 años. De los hipertensos, el 75\% tiene DM mayor a 15 años desde su diagnóstico.

EI SM estuvo presente en 8/64 pacientes (12,5\%), mitad hombres y mitad mujeres. Sin embargo, se destaca que esta variable probablemente subestime la prevalencia dado que en 14 pacientes $(21,9 \%)$ no se contó con los datos antropométricos.

$20 / 64$ pacientes $(31,3 \%)$, además de ser diabéticos, asociaban un componente de SM.

En el total de pacientes que se pudo aplicar la fórmula de CG, el $44 \%$ de ellos presentó valores $<8,77$. No pudo calcularse la CG en 13 casos por no contar con las medidas antropométricas, en 11 casos no se pudo determinar por $\mathrm{HbA} 1 \mathrm{c} \geq 11,4$ y en un caso no 
se logró determinar la A1c.

Al comparar los subgrupos según $\mathrm{CG}$, se encontró que en el subgrupo de $C G<8,77$ ( $n=17$ ), se registró un caso de SM (6\%), 6 pacientes asociaron otro componente de SM y 10 no tenían SM (59\%). Los pacientes que asocian al menos un componente del SM alcanzaron una prevalencia de $35 \%$. En el otro extremo, el subgrupo con $C G \geq 8,77(\mathrm{n}=22)$ no registró casos de SM; 3 pacientes asociaban al menos otro componente del SM (prevalencia 14\%) y 19 casos $(86 \%)$ no presentaban ningún elemento de SM excepto la DM que define a esta población.

Se destaca entonces que la prevalencia de dos componentes de SM (Diabetes y otro componente adicional) fue superior (35\%) en el subgrupo con $C G<8,77$ vs el subgrupo con $C G \geq 8,77(14 \%)$, sin ser estadísticamente significativo (Test chi cuadrado con corrección de Yates, $p=0,18$ ).

En relación a control metabólico, encontramos que el promedio de $\mathrm{HbA} 1 \mathrm{c}$ fue de 9,3 $\pm 2,0$ (DE) para la población estudiada. $12,5 \%$ presentaba $\mathrm{HbA} 1 \mathrm{c} \leq 7,5$, valor considerado óptimo para población adolescente en general según recomendación ISPAD (30), sin dejar de tener en cuenta que es un valor que debe individualizarse. $8 \%$ presentaba $\mathrm{HbA} 1 \mathrm{c} \leq 7$, siendo todos pacientes menores de 18 años. Los pacientes con $\mathrm{HbA} 1 \mathrm{c} \leq 7,5$ presentaban un tiempo de evolución de la enfermedad de 7,5 años, menor que el observado para la población con $\mathrm{HbA} 1 \mathrm{c}>$ 7,5 (12,9 años). Al relacionar $\mathrm{HbA} 1 \mathrm{c}$ con $\mathrm{CG}$, encontramos que el subgrupo con $\mathrm{CG}<8,77$ tuvo promedio $\mathrm{HbA1c}$ de 9,12, mayor que el subgrupo de $\geq 8,77$ ( $\mathrm{HbA} 1 \mathrm{c} 8,7$ promedio) (diferencia no significativa).

\section{DISCUSIÓN}

Se asiste a una población de pacientes con DM1 procedente fundamentalmente del área metropolitana de Montevideo; jóvenes, sin clara predominancia entre géneros.

Más de la mitad de esta población presenta un tiempo de evolución desde el diagnóstico de DM de hasta 10 años, similar a los encontrados en otras bibliografías. Si bien predomina el normopeso, es preocu- pante la alta prevalencia de sobrepeso y obesidad (38\%). En relación a SM, no se registró diferencia entre ambos sexos, en contraposición con la literatura que muestra predominancia del sexo femenino. La prevalencia de SM encontrada en nuestro trabajo es menor que la reportada en la bibliografía consultada; esto podría explicarse dada las características demográficas de nuestra serie (predominancia de adolescentes y adultos jóvenes) $(8,16,19)$.

Dentro de SM el componente más prevalente fue el HDL de riesgo (25\%), al igual que otras series $(16,19)$ seguido del perímetro de cintura de riesgo (20\%), ambos predominantes en mujeres jóvenes. En tercer lugar se encuentra la hipertrigliceridemia (16\%) que a diferencia de los anteriores, predominó en hombres jóvenes. Con respecto a la HA, fue el componente de SM con menor prevalencia (10\%) encontrándose fundamentalmente en adultos maduros y/o mayores (67\%). Creemos que la explicación de este hecho también podría corresponder a la edad de la población estudiada (población joven), ya que otras series con población de mayor edad encuentran mayor prevalencia de HA $(16,19)$.

Si bien la prevalencia de SM (3 de 5 criterios) fue baja $(12,5 \%)$, no lo fue la presencia de sus componentes en forma aislada (31,3\%). Estas cifras totalizan $44 \%$, porcentaje no despreciable en población de diabéticos tipo 1 con RI (Diabetes Doble).

Se registró una relación entre SM y CG baja, sin ser estadísticamente significativa.

La CG descendida fue $44 \%$; menor que la reportada por Sanhueza, que encuentra $75 \%$.

Sin embargo, al igual que este autor, no se encontró concordancia entre la frecuencia de SM $(12,5 \%)$ y la presencia de Resistencia Insulínica cuantificada por la fórmula de CG. Cabe destacar que las poblaciones estudiadas difieren en la edad de inclusión (Sanhueza incluye mayores de 18 años y en nuestro trabajo el $31 \%$ de la población tiene $\leq 18$ años) y en el promedio etario (en el estudio de Sanhueza es mayor). Se encontró un valor promedio de $\mathrm{HbA} 1 \mathrm{c}$ de $9,3 \%$, superior a la reportada por otros investigadores. El porcentaje de pacientes que se encuen- 
tran en rango meta de $\mathrm{HbA} 1 \mathrm{c}$ es bajo, sabiendo que este es un parámetro que debe individualizarse. Los pacientes que alcanzaron $\mathrm{HbA} 1 \mathrm{c}$ meta, presentaban menor tiempo de evolución de la enfermedad. Los pacientes con CG baja tuvieron promedios de $\mathrm{HbA} 1 \mathrm{c}$ superiores al resto. En $17 \%$ de los pacientes, no se pudo aplicar la fórmula de $\mathrm{CG}$ por $\mathrm{HbA1c} \geq$ $11,4 \%$, similar a la reportada por Sanhueza $(8,11)$.

\section{CONCLUSIONES}

Se estudió una población con DM1 que asocia SM: "Diabetes Doble"; la misma constituye un desafío para el equipo asistencial, ya que a la complejidad propia del manejo de la DM1, se asocia la exposición a FRCV desde etapas tempranas de la vida. La prevención y la detección precoz de los mismos incidirían favorablemente en la prevención o retraso de la aparición en el tiempo de la enfermedad cardiovascular, principal causa de muerte de esta población.

\section{RECOMENDACIONES}

Nos encontramos ante una población con un alto riesgo de desarrollar Enfermedad Cardiovascular, principal causa de muerte global y particularmente en DM. Estos hechos cobran mayor jerarquía si consideramos que se trata de población joven con alta expectativa de vida. Esto implica centrar la atención de estos pacientes no solo en el control metabólico, sino también en la detección precoz de FR CV, así como en su manejo agresivo y temprano.

En relación a la documentación de datos clínicos, al analizar las historias, (fuente secundaria) surge como evidente el subregistro de datos, tanto sociodemográficos como antropométricos. A tales efectos podría ser de utilidad la creación de una ficha pre establecida que contenga los datos mencionados, a ser llenados al ingreso al servicio por el personal integrante del equipo asistencial.

\section{REFERENCIAS}

(1) Guías ALAD sobre el Diagnóstico, Control y Tratamiento de la Diabetes Mellitus Tipo 2 con Medicina Basada en Evidencia. Revista de la ALAD 2013. 142 p. Disponible en: http://endosuem.org.uy/wp-content/ uploads/2015/05/GUIAS-ALAD-2013.pdf [Consulta 20/03/2018]

(2) Standards of Medical Care in Diabetes 2017. Diabetes Care 2017; 40(Suppl 1):S1-S132.

(3) Ahlqvist E, Storm $P$, Käräjämäki $A$, Martinell $M$, Dorkhan M, Carlsson A, et al. Novel subgroups of adult-onset diabetes and their association with outcomes: a data-driven cluster analysis of six variables. Lancet Diabetes Endocrinol 2018; 6(5):361-369. doi:10.1016/S2213-8587(18)30051-2

(4) Leslie RD, Palmer J, Schloot NC, Lernmark A. Diabetes at the crossroads: relevance of disease classification to pathophysiology and treatment.

Diabetologia 2016; 59(1):13-20.

doi: $10.1007 / s 00125-015-3789-z$

(5) Expert Panel on Detection, Evaluation, and Treatment of High Blood Cholesterol in Adults. Executive Summary of the Third Report of the National Cholesterol Education Program (NCEP) Expert Panel on Detection, Evaluation, and Treatment of High Blood Cholesterol in Adults (Adult Treatment Panel III). JAMA 2001; 285(19):2486-2497.

(6) James PA, Oparil S, Carter BL, Cushman WC, Dennison-Himmelfarb C, Handler J, et al. 2014 evidence-based guideline for the management of high blood pressure in adults: Report from the panel members appointed to the Eighth Joint National Committee (JNC 8). JAMA 2014; 311(5):507-20. doi: 10.1001/jama.2013.284427

(7) Bulum T, Duvnjak L. Insulin resistance in patients with type 1 diabetes: relationship with metabolic and inflammatory parameters. Acta Clin Croat 2013; 52(1):43-51. 
(8) Sanhueza L, Concha L, Durruty P, Rubio C, Wolff C, García de los Ríos M. Diabéticos tipo 1 portadores de síndrome metabólico: cuantificación de la resistencia a la insulina.

Rev chil endocrinol diabetes 2014; 7(3):89-93.

(9) Donga E, van Dijk M, van Dijk JG, Biermasz NR, Lammers GJ, van Kralingen $\mathrm{K}$, et al. Partial sleep restriction decreases insulin sensitivity in type 1 diabetes. Diabetes Care 2010; 33(7):1573-7.

doi: $10.2337 / \mathrm{dc} 09-2317$

(10) Schauer IE, Snell-Bergeon JK, Bergman BC, Maahs DM, Kretowski A, Eckel RH, et al. Insulin resistance, defective insulin-mediated fatty acid suppression, and coronary artery calcification in subjects with and without type 1 diabetes: The CACTI study.

Diabetes 2011; 60(1):306-14. doi: 10.2337/db10-0328

(11) Epstein EJ, Osman JL, Cohen HW, Rajpathak SN, Lewis O, Crandall JP. Use of the estimated glucose disposal rate as a measure of insulin resistance in an urban multiethnic population with type 1 diabetes. Diabetes 2013; 36(8):2280-5.

doi: $10.2337 / \mathrm{dc} 12-1693$

(12) Girgis CM, Scalley BD, Park KE. Utility of the estimated glucose disposal rate as a marker of microvascular complications in young adults with type 1 diabetes. Diabetes Res Clin Pract 2012; 96(3):e70-2. doi: 10.1016/j.diabres.2012.02.004

(13) Pop A, Clenciu D, Anghel M, Radu S, Socea B, Mota $\mathrm{E}$, et al. "Insulin resistance is associated with all chronic complications in type 1 diabetes".

J Diabetes 2016; 8(2):220-8.

doi: $10.1111 / 1753-0407.12283$

(14) Pambianco G, Costacou T, Orchard TJ. The prediction of major outcomes of type 1 diabetes: a 12 year prospective evaluation of three separate definitions of the metabolic syndrome, and their components and estimated glucose disposal rate: the Pittsburg Epidemiology of Diabetes Complications Study experience.

Diabetes Care 2007; 30(5):1248-54.
(15) Merger SR, Kerner W, Stadler M, Zeyfang A, Jehle $P$, Müller-Korbsch $M$, et al. Prevalence and comorbidities of double diabetes.

Diabetes Res Clin Pract 2016; 119:48-56.

doi: 10.1016/j.diabres.2016.06.003

(16) Ferreira Hermosillo A, Vargas Ortega G, González Virla B, Mercado Atri M, Molina Ayala M. Prevalencia del síndrome metabólico (SM) en pacientes diabeticos tipo 1.

Gac Med Mex 2012; 148(2):137-43.

(17) Cleland SJ, Fisher BM, Colhoun HM, Sattar N, Petrie JR. 2013. Insulin resistance in type 1 diabetes: what is "double diabetes" and what are the risks?. Diabetología 2013; 56:1462-1470.

(18) Teupe B, Bergis K. Epidemiological evidence for “double diabetes”. Lancet 1991; 337(8737):361-2.

(19) Chillarón JJ, Goday A, Flores-Le-Roux JA, Benaiges D, Carrera MJ, Puig J, et al. Estimated glucose disposal rate in assessment of the metabolic syndrome and microvascular complications in patients with type 1 diabetes.

J Clin Endocrinol Metab 2009; 94(9):3530-4.

doi: 10.1210/jc.2009-0960

(20) The DCCT Research Group. The relationship of glycemic exposure $(\mathrm{HbA} 1 \mathrm{c})$ to the risk of development and progression of retinopathy in the Diabetes Control and Complications Trial.

Diabetes 1995; 44(8):968-983.

(21) Atala Dib S. Insulin resistance and metabolic syndrome in type 1 diabetes mellitus.

Arq Bras Endocrinol Metab 2006; 50(2):250-263.

(22) Báez MS, Novik V, Alegría F, Cardemil F, Riveros $\mathrm{R}$, Bofill L. Síndrome metabólico en un grupo de pacientes diabéticos tipo 1. ¿Una nueva variedad de diabetes? Rev Méd Chile 2009; 137(7):888-893. http://dx.doi.org/10.4067/S0034-98872009000700004 
(23) Momesso DP, Bussade I, Lima GA, Fonseca LP, Russo LA, Kupfer R. Body composition, metabolic syndrome and insulin resistance in type 1 diabetes mellitus.

Arq Bras Endocrinol Metabol 2011; 55(3):189-93.

(24) Uribe-Wiechers AC, Janka-Zires M, AlmedaValdés $P$, López-Gutiérrez J, Gómez-Pérez FJ. Albuminuria and Glomerular Filtration Rate in Individuals with Type 1 Diabetes Mellitus: Contribution of Metabolic Syndrome.

Rev Invest Clin 2015; 67(4):266-272.

(25) Timar R, Timar B, Degeratu D, Serafinceanu C, Oancea C. Metabolic syndrome, adiponectin and proinflammatory status in patients with type 1 diabetes mellitus. J Int Med Res 2014; 42(5):1131-8.

doi: $10.1177 / 0300060514541829$

(26) Pisabarro R, Gutiérrez M, Bermúdez C, Prendez D, Recalde A, Chaftare $Y$, et al. Segunda Encuesta Nacional de Sobrepeso y Obesidad (ENSO 2) adultos (18-65 años o más).

Rev Méd Urug 2009; 25(1):14-26.

(27) Ferrero R, García MV. Encuesta de prevalencia de la diabetes en Uruguay. Primera fase: Montevideo Año 2004.

Arch Med Int 2005; 27:7-12.

(28) Uruguay. Ministerio de Salud Pública, Dirección General de la Salud, División Epidemiología, 1a Encuesta Nacional de Factores de Riesgo de Enfermedades Crónicas No Transmisibles. 33 p. Disponible en: file:///C:/Users/pc/Desktop/1er_enfrecnt_2006_1. pdf [Consulta 13/04/2018].

(29) Uruguay. Ministerio de Salud Pública. Programa de Prevención de Enfermedades no Transmisibles. 2da. Encuesta Nacional de Factores de Riesgo de Enfermedades no Transmisibles. 44 p. Disponible en: file:///C:/Users/pc/Desktop/2DA_ENCUESTA_NACIONAL_final2_digital.pdf [Consulta 20/03/2018].
(30) Craig ME, Hattersley A, Donaghue K. ISPAD Clinical Practice Consensus Guidelines 2009 Compendium. Definition, epidemiology and classification of diabetes in children and adolescent. Pedriatric Diabetes 2009; 10(Suppl 12):3-12. 